\title{
Clinical Outcomes of Electrodiagnostic Testing Conducted in Primary Care
}

\author{
Mark M. Shepherd, MD
}

Background: Focal and generalized neuropathies, such as carpal tunnel syndrome and diabetic peripheral neuropathy, have high prevalence among the US population. Primary care physicians are increasingly using computerized electrodiagnostic (EDX) testing to evaluate these patients. The impact of this diagnostic modality on physician diagnostic reasoning and patient management has not been directly studied.

Methods: Consecutive computerized EDX tests conducted in a primary care clinic were prospectively evaluated using a diagnostic before-and-after study design. The pretest diagnosis and patient management plan were noted before EDX testing. Five clinical outcomes were assessed after the test: (1) test abnormality, (2) confirmation of pretest diagnosis, (3) change in pretest diagnosis, (4) expansion of pretest diagnosis, and (5) change to patient management plan.

Results: One hundred computerized EDX tests were performed among 85 patients. A total of 88 tests were abnormal. The pretest diagnosis was confirmed in 59 tests. The pretest diagnosis was changed by 14 tests and it was expanded by 18 tests. The computerized EDX tests triggered a change in clinical management in 30 cases, with referral to orthopedic surgery and metabolic workup for polyneuropathy as the most common changes.

Conclusions: Computerized EDX testing was clinically valuable in patients with moderate to high pretest probability of common neuropathies such as carpal tunnel syndrome and diabetic polyneuropathy. In approximately one third of cases, the test led to a change in clinical management. ( $\mathrm{J}$ Am Board Fam Med 2010;23:584-590.)

Keywords: Nerve Conduction, Clinical Outcomes, NC-stat, Primary Care

Focal and generalized neuropathies have become increasingly common during the past several decades. A study of carpal tunnel syndrome ${ }^{1}$ among the general population reported a $14.4 \%$ prevalence of symptoms in the median nerve distribution and a $2.7 \%$ prevalence of electrodiagnostically confirmed disease. In a population-based study of diabetes, ${ }^{2} 22 \%$ of participants had moderate to severe peripheral neuropathy. Another large study recently showed that peripheral sensory neuropathies

This article was externally peer reviewed.

Submitted 22 March 2010; revised 21 May 2010; accepted 26 May 2010.

From the Intermountain Medical Group, Salt Lake City, UT.

Funding: none.

Conflict of interest: The principle investigator has no financial or other affiliation with NeuroMetrix, Inc. This study was initiated, designed, and completed independently of the company.

Corresponding author: Mark M. Shepherd, MD, Intermountain Medical Group, Bryner Clinic, 525 East 100 South, Suite 500, Salt Lake City, UT 84102 (E-mail: Mark.Shepherd@imail.org). are common, with a prevalence of $31 \%$ among primary care patients aged 65 years or older. ${ }^{3}$ Because of the high prevalence of focal and generalized neuropathies, family and internal medicine physicians may now be encountering neuropathies at higher rates and earlier in their natural history.

Although a neurological examination is central to the workup of patients with suspected neuropathies, it may not have adequate diagnostic accuracy. ${ }^{4-6}$ Therefore, objective confirmation of a neuropathy and grading of severity may benefit patient management. ${ }^{7}$ Nerve conduction studies (NCSs) provide the most objective and reliable diagnostic assessment of neuropathies. ${ }^{8,9}$ As such, expanded access to NCSs has the potential to improve clinical outcomes. ${ }^{7}$ However, constraints on the availability of NCSs have probably limited its usage, ${ }^{7,10}$ particularly within primary care and early in the episode of care. In response, technological advances in computerized electrodiagnostic (EDX) instrumentation and methods have been introduced during the past 10 years. These innovations include nerve-specific electrode 
arrays, computer-aided data acquisition, and decision support. ${ }^{11}$ Because this technology standardizes and automates NCSs up to the point of diagnostic interpretation (which is performed by the physician), data quality is essentially independent of clinical setting. This property has led to use of computerized EDX instrumentation in multicenter drug registration trials ${ }^{12}$ and large-scale epidemiologic studies. ${ }^{13,14}$ Further, because of inherent consistency, the accuracy of the results as assessed in controlled clinical studies should translate to clinical practice. Diagnostic accuracy, with traditional EDX instrumentation or neuroimaging (for radiculopathy) as reference standards, has been reported for carpal tunnel syndrome, ${ }^{15-17}$ upper-extremity nerve function in symptomatic patients, ${ }^{18-20}$ diabetic peripheral neuropathy, ${ }^{21,22}$ lumbosacral radiculopathy, ${ }^{23-26}$ and lower-extremity nerve function in symptomatic patients. ${ }^{27}$

Computerized EDX studies are now commonly provided by primary care physicians. Several large observational studies have evaluated the use of NCSs within primary care. ${ }^{28,29}$ These studies showed that patient selection was appropriate and that computerized EDX testing results had the potential to alter pretest diagnoses. However, these studies did not specifically evaluate the clinical impact of testing. ${ }^{30}$ The objective of this study was to measure changes in physician diagnostic reasoning and patient management resulting from computerized EDX testing in primary care.

\section{Methods}

This study was designed as a prospective diagnostic before-and-after ${ }^{31}$ examination of the impact of computerized $\mathrm{EDX}^{11}$ testing on physician diagnostic reasoning and patient management. All patients were seen at a single clinic staffed by a group of 5 outpatient-only, primary care-based internists, all board certified in internal medicine. The clinic is located in an urban setting; however, it also draws patients from outlying suburban and rural areas. A clinical diagnosis was made from the symptoms and physical examination, and then computerized EDX testing was conducted when deemed medically valuable during the course of routine clinical care. Specific screening or selection criteria were not applied. A total of 165 consecutive computerized EDX tests conducted during a 13-month period were evaluated. The first 65 studies were evaluated retrospectively by chart review and the results were used to refine the outcome definitions and study procedures for the prospective phase. The next 100 studies were evaluated prospectively and formed the study data set. Both the retrospective chart reviews and the prospective assessment of outcomes were performed by the principle investigator (MMS). The computerized EDX testing was performed by trained clinical staff with supervision and clinical interpretation by the principle investigator. The outcome data were collected as part of a technology assessment to determine whether computerized EDX testing within primary care was feasible and clinically useful. This study was performed in accordance with institutional review board policies at the parent institution.

All of the EDX studies were performed using a single computerized EDX device (NC-stat; NeuroMetrix, Inc., Waltham, MA). This device was used as clinically indicated. Device operation has been described previously. ${ }^{11}$ In brief, this device comprises nerve-specific electrode arrays that standardize nerve conduction measurements, data acquisition, analysis instrumentation, and computerized decision support. The device outputs nerve conduction parameter values, a comparison of those values to normative data, and a list of neuropathy types that are consistent with the measurements. This latter output is similar to the analyses provided by modern electrocardiography devices. ${ }^{32}$ The algorithms are proprietary but generally based on evidence-based practice parameters, ${ }^{11}$ such as those published for carpal tunnel syndrome ${ }^{8}$ and peripheral neuropathy. ${ }^{9}$

Before initiating the study, 5 clinical outcomes were defined. The first was whether or not the EDX test was abnormal. A test was defined as abnormal if the computerized EDX device identified any nerves as having abnormal nerve conduction measurements, regardless of whether the physician regarded those abnormalities as clinically significant. The second was whether the computerized EDX output confirmed the pretest diagnosis. The third was whether the computerized EDX test changed the physician's pretest diagnosis. This was defined as a complete change from the suspected pretest diagnosis to another diagnosis. The fourth was whether the computerized EDX test expanded the pretest diagnosis by adding a previously unsuspected diagnosis. Asymptomatic conditions were included if they were moderate or severe 
by NCS criteria. The fifth was whether the plan of care was altered by the computerized EDX test. This was defined as ordering a diagnostic test (eg, imaging, blood tests), implementing a change in therapy (eg, use of splinting), or initiating a referral (eg, orthopedic surgery, podiatry, neurology) because of the computerized EDX results. The type of change was recorded. During the prospective phase the pretest diagnosis and treatment plan were noted by the principle investigator before the computerized EDX test was performed. The diagnosis after the EDX test was generally based on the $\mathrm{NC}$-stat output unless the principle investigator disagreed with the analyses based on the clinical assessment. This occurred most often when nerve conduction measurements were technically normal (ie, within 2 SDs of the mean) but at the high end of the reference range, which indicated consistency but not absolute agreement with the clinical diagnosis. These instances were logged and are reported in the Results section, below. The diagnosis after the test and changes to the treatment plan were determined within several days of receipt of the computerized EDX results.

The outcome data were primarily analyzed using descriptive statistics. The homogeneity of outcomes among the pretest diagnostic categories was evaluated using the contingency $\chi^{2}$ test. Homogeneity was rejected at the $P<.1$ significance level. 95\% CIs were determined for each of the clinical outcomes.

\section{Results}

A total of 100 computerized EDX tests from 85 different patients performed during a 10-month period (February through November, 2007) were analyzed prospectively. Among the patients evaluated, $56.6 \%$ were women; the mean age was $60.0 \pm$
16.8 years, with $53.0 \% \geq 65$ years; mean body mass index was $30.3 \pm 7.1 \mathrm{~kg} / \mathrm{m}^{2}$, with $21.7 \%$ considered severely or morbidly obese $\left(\geq 35 \mathrm{~kg} / \mathrm{m}^{2}\right)$.

Table 1 lists clinical outcomes by pretest diagnosis. The most common specific diagnoses were carpal tunnel syndrome and diabetic polyneuropathy. In 20 tests, the pretest diagnosis was both lumobsacral radiculopathy and polyneuropathy. On average, $5.9 \pm 1.6$ nerves (motor or sensory) were tested per study, and $3.3 \pm 2.6$ nerves were abnormal. Eighty-eight of 100 tests were abnormal as defined by the presence of at least one abnormal nerve. The results of one test were normal, but the patient had significant clinical symptoms and was immediately referred for magnetic resonance imaging. In 7 tests all of the nerves relevant to the pretest diagnosis (eg, median nerve for carpal tunnel syndrome) were normal; however, there was no change in diagnosis after the test. In other words, the pretest diagnosis was retained based on clinical findings. Mild NCS abnormalities without clinical significance were identified in 34 tests. In many cases these findings were incidental to abnormalities that confirmed the pretest diagnosis (eg, mild ulnar abnormalities in carpal tunnel syndrome).

In 59 tests (95\% CI, 49-68), the pretest diagnosis was confirmed by computerized EDX testing. This outcome ranged from $36.4 \%$ of tests for nonspecific polyneuropathies to $76.5 \%$ of tests for carpal tunnel syndrome (see Table 1). The distribution of outcomes was heterogeneous. In an additional 14 tests, the results were within normal limits but near the abnormality threshold and therefore were consistent with the pretest diagnosis. Seven tests were inadequate because of incomplete data or technical difficulties that prevented

Table 1. Clinical Impact of Computerized Electrodiagnostic Testing $(\mathbf{n}=\mathbf{1 0 0})$

\begin{tabular}{lccccr}
\hline & & \multicolumn{3}{c}{ Impact on Pretest DX (\%) } \\
Pretest DX & $\mathrm{n}$ & Confirmed DX* & Changed DX & Expanded DX & Changed Management \\
\hline Carpal tunnel syndrome & 34 & 76.5 & 0.0 & 20.6 & 41.2 \\
Diabetic PN & 18 & 55.6 & 5.6 & 16.7 & 22.2 \\
Other PN & 11 & 36.4 & 27.3 & 18.2 & 27.3 \\
LSR & 17 & 41.2 & 17.6 & 5.9 & 29.4 \\
LSR or PN & 20 & 60.0 & 35.0 & 25.0 & 20.0 \\
Total [n (95\% CI)] & 100 & $59(49-68)$ & $14(8-22)$ & $18(12-27)$ & $30(22-40)$ \\
\hline
\end{tabular}

*Homogeneity among the pretest diagnostic categories rejected by contingency $\chi^{2}$ test at $P<.1$.

DX, diagnosis; LSR, lumbosacral radiculopathy; PN, polyneuropathy. 
evaluation of the pretest diagnosis. The remaining 27 tests did not confirm the pretest diagnosis.

A total of 14 computerized EDX tests (95\% CI, $8-22$ ) changed the pretest diagnosis. In those tests with a pretest diagnosis of carpal tunnel syndrome, there were no instances of the test changing the pretest diagnosis. Conversely, when the pretest diagnosis was lumbosacral radiculopathy and polyneuropathy, $35 \%$ of tests changed the diagnosis. This distribution was statistically significant (ie, homogeneity rejected). In 18 tests (95\% CI, 1227), computerized EDX tests expanded the pretest differential diagnosis with one or more additional diagnosis. For example, several patients with putative carpal tunnel syndrome were identified as potentially also having an ulnar neuropathy or polyneuropathy. In aggregate, 31 computerized EDX tests either changed or expanded the pretest diagnosis.

The computerized EDX tests triggered a change in the clinical management plan in 30 cases $(95 \%$ CI, 22-40). The distribution of rates among the diagnostic categories was limited and heterogeneity was rejected. A summary of the changes in patient management is provided in Table 2. The most common changes were referral to orthopedic surgery and metabolic workup for polyneuropathy. Other changes included referral to a podiatrist, further diagnostic testing, and specific treatments. Among the 30 patients with altered management plans, the computerized EDX test confirmed the pretest diagnosis of $24(80 \%)$.

\section{Discussion}

This prospective diagnostic before-and-after study ${ }^{31}$ demonstrated that computerized EDX testing in a

Table 2. Changes in Clinical Management Resulting from Computerized Electrodiagnostic Testing $(n=30)$

\begin{tabular}{lc}
\hline Change & Count* \\
\hline Orthopedic referral & 9 \\
Metabolic work-up for PN & 5 \\
NCS work-up for CTS & 4 \\
Prescribe splints & 4 \\
Change medication & 4 \\
Podiatry referral & 3 \\
Order MRI & 2 \\
\hline
\end{tabular}

*Sum is $>30$ because one study had 2 management changes. PN, polyneuropathy; NCS, nerve conduction studies; CTS, carpal tunnel syndrome; MRI, magnetic resonance imaging. primary care setting was clinically useful. Changes in clinical management were triggered in $30 \%$ of tests. This rate is comparable to the $30 \%$ to $50 \%$ reported for electrocardiography ${ }^{33,34}$ and spirometry ${ }^{35}$ use in primary care. In this study, orthopedic referrals and metabolic workups for polyneuropathy were the most common change, and they generally followed confirmation of advanced carpal tunnel syndrome or detection of polyneuropathy. No patients were immediately referred to neurology; however, it is possible that neurology referrals occurred at a later time. The number of magnetic resonance imaging studies prompted by computerized EDX testing was small $(n=2)$. Treatment alterations were made in 8 instances as a result of testing. This study did not explicitly record clinical outcomes resulting from patient management decisions triggered by computerized EDX testing. However, one interesting example identified during the retrospective phase was a patient with a pretest diagnosis of polyneuropathy who was identified as having a possible lumbosacral radiculopathy. Because of the clinical discordance, the patient had a magnetic resonance imaging study, which showed a spinal cord tumor. There were also patients for whom computerized EDX testing suggested that prior diagnostic procedures, such as blood tests among patients with lumbosacral radiculopathy, may have been unnecessary. These instances were not included in any outcome measure.

Almost $90 \%$ of tests had at least one abnormal nerve. This rate is similar to prior reports of multicenter observational studies. ${ }^{28,29}$ The high rate of abnormal results implies that computerized EDX testing was used among patients with a high pretest probability of nerve dysfunction. In this situation, confirmation of the pretest diagnosis raises the probability of disease after the test to a high level, and thus gives the physician confidence that the patient has the condition. ${ }^{36}$ For example, if a patient has a pretest probability of carpal tunnel syndrome of .5 based on their clinical examination, then a positive study raises the probability to $>.90$ because computerized EDX testing has been shown to have a positive likelihood ratio of $>10$ for detecting median neuropathy at the wrist. ${ }^{15,16}$ In approximately $60 \%$ of tests, computerized EDX testing confirmed the physician's pretest diagnosis. Physicians attribute significant value to diagnostic results that reassure them that they've made a correct diagnosis. ${ }^{30}$ Consistent with that principle, 
computerized EDX testing confirmed the pretest diagnosis in $80 \%$ of studies that also led to a change in patient management.

The rates of confirmed and changed pretest diagnoses were not homogeneous. This is not surprising; the pretest diagnostic categories represented a number of distinct clinical entities with different pathologic origins, patient characteristics, and clinical presentations. The fairly uniform rate of changes to patient management, despite variations in diagnostic output, is therefore interesting. It may be valuable to examine the clinical profile of patients who most benefit from computerized EDX testing in future studies.

Despite 5 decades of use, few studies in the literature have assessed the impact of EDX testing on physician diagnostic reasoning and patient management. ${ }^{37,38}$ Table 3 summarizes clinical outcomes common to this study and 4 prior studies that reported results of referrals to academic EDX laboratories. As shown in Table 3, there are substantial differences in the patient populations, which must be considered when comparing outcomes. The percentage of abnormal tests was higher in this study than for referral EDX testing. In addition, referral testing led to a change in patient management in a slightly higher percentage of cases. Tests conducted on patients referred to EDX laboratories seem less likely to confirm the pretest diagnosis than those conducted in a primary care setting. The reason for this difference is unclear, but it may reflect conservative patient selection for computerized EDX testing, whereas patients referred to academic centers are probably complex with a higher level of diagnostic uncertainty before a test. This type of triage between relatively straightforward patients being evaluated within primary care and referral of complex pa- tients seems clinically reasonable and may optimize use of health care resources.

Several limitations of the methodology should be considered when interpreting the results of this study. ${ }^{31}$ This study was performed at one primary care clinic by one clinician. Although the case mix and rate of abnormalities were similar to previous reports from large, multicenter observational studies, ${ }^{28,29}$ it is possible that the results will not generalize to other primary care clinics. One of the inherent limitations of diagnostic before-and-after studies is that assessments before and after tests by the same clinicians for the same patients may be interrelated, and therefore the independent impact of the diagnostic test cannot be determined. ${ }^{31}$ However, this is not necessarily a deficiency because real-world clinical practice is a process of aggregating information to arrive at a clinical conclusion. Furthermore, because this analysis was independent and was conducted within the framework of a technology assessment to determine whether computerized EDX testing was feasible and useful, it was unlikely that the lack of pretest blinding substantially biased the results in a favorable direction. Potential misclassifications by computerized EDX testing were not evaluated by comparing the results to a reference standard (eg, referral to a traditional EDX laboratory) or by long-term follow-up to confirm the EDX results. There is no gold standard for assessment of neuropathies; traditional EDX laboratories and other diagnostic methods are associated with misclassifications. ${ }^{39-42}$ Long-term follow-up may be useful for confirming diagnostic accuracy but, with narrow exceptions, ${ }^{43}$ it has not been performed for traditional EDX studies; therefore, established methods and benchmarks do not exist. Nevertheless, it is unlikely that computerized EDX testing

Table 3. Published Studies of Electrodiagnostic Clinical Outcomes

\begin{tabular}{lccccccccc}
\hline & & \multicolumn{3}{c}{ Diagnosis Before Test (\%) } & & \multicolumn{3}{c}{ Diagnosis After Test (\%) } \\
Reference & Studies $(\mathrm{n})$ & CTS & LSR/PN & Other* & & Abnormal & Confirmed & Changed Plan \\
\hline Current study & 100 & 34.0 & 66.0 & 0 & & 88.0 & 59.0 & 30.0 \\
Kothari et al, 1995 & 126 & 26.0 & 33.0 & 41.0 & & 60.3 & 31.7 & NA \\
Kothari et al, 1998 & 100 & 17.5 & 27.0 & 55.6 & & 78.0 & 49.0 & 46.0 \\
Lo et al, 2002 & 348 & 100 & 0 & 0 & & 56.3 & 48.6 & NA \\
Cho et al, 2004 & 44 & 0 & 100 & 0 & & 81.8 & 38.6 & 41.0 \\
\hline
\end{tabular}

${ }^{*}$ Cervical radiculopathy; brachial and lumbosacral plexopathy; focal neuropathies (radial, ulnar, peroneal, proximal median, tibial); motor neuron disease; myasthenia gravis; and myopathy.

CTS, carpal tunnel syndrome; LSR, lumbosacral radiculopathy; PN, polyneuropathy. 
misclassified many patients because diagnostic accuracy of this methodology has been previously established. ${ }^{15,22,23}$ Finally, this study assessed the impact of computerized EDX testing on physician diagnostic reasoning and patient management, but the specific benefit of these changes to patient outcomes was not measured. An answer to this question would probably require a prospective randomized controlled trial, ${ }^{30}$ which have rarely been performed for even widely used diagnostic procedures. In this study, the primary impact of computerized EDX testing was a specialist referral or an additional diagnostic workup. Assuming that the EDX test results were correct, these decisions probably resulted in incremental clinical attention to relevant patients. However, a definitive demonstration of this fact may require additional types of studies.

The results of this prospective, diagnostic, before-and-after study suggest that computerized EDX testing is clinically valuable for patients with moderate to high pretest probability of common neuropathies such as carpal tunnel syndrome and diabetic polyneuropathy. Approximately $60 \%$ of computerized EDX tests confirmed the physician's pretest diagnosis and approximately $30 \%$ changed or expanded the diagnosis. This impact on diagnostic thinking led to a change in clinical management after $30 \%$ of tests.

The author acknowledges assistance with manuscript preparation from Shai N. Gozani, MD, PhD, of NeuroMetrix Inc.

\section{References}

1. Atroshi I, Gummesson C, Johnsson R, Ornstein E, Ranstam J, Rosen I. Prevalence of carpal tunnel syndrome in a general population. JAMA 1999;282: 153-8.

2. Abbott CA, Carrington AL, Ashe H, et al. The North-West Diabetes Foot Care Study: incidence of, and risk factors for, new diabetic foot ulceration in a community-based patient cohort. Diabet Med 2002;19:377-84.

3. Mold JW, Vesely SK, Keyl BA, Schenk JB, Roberts $M$. The prevalence, predictors, and consequences of peripheral sensory neuropathy in older patients. J Am Board Fam Pract. 2004;17:309-18.

4. Katz JN, Stirrat CR, Larson MG, Fossel AH, Eaton HM, Liang MH. A self-administered hand symptom diagram for the diagnosis and epidemiologic study of carpal tunnel syndrome. J Rheumatol 1990;17:1495-8.

5. Descatha A, Dale AM, Franzblau A, Coomes J, Evanoff B. Diagnostic strategies using physical examination are minimally useful in defining carpal tunnel syndrome in population-based research studies. Occup Environ Med 2010;67:133-5. Epub 2009 Oct 22.

6. Vinik AI, Kong X, Megerian JT, Gozani SN. Diabetic nerve conduction abnormalities in the primary care setting. Diabetes Technol Ther 2006;8:654-62.

7. Perkins BA, Bril V. Diabetic neuropathy: a review emphasizing diagnostic methods. Clin Neurophysiol 2003;114:1167-75.

8. Jablecki CK, Andary MT, Floeter MK, et al. Practice parameter: electrodiagnostic studies in carpal tunnel syndrome. Report of the American Association of Electrodiagnostic Medicine, American Academy of Neurology, and the American Academy of Physical Medicine and Rehabilitation. Neurology 2002;58: 1589-92.

9. England JD, Gronseth GS, Franklin G, et al. Distal symmetrical polyneuropathy: definition for clinical research. Muscle Nerve 2005;31:113-23.

10. Duncan KH, Lewis RC Jr, Foreman KA, Nordyke MD. Treatment of carpal tunnel syndrome by members of the American Society for Surgery of the Hand: results of a questionnaire. J Hand Surg Am 1987;12:384-91.

11. Gozani SN, Fisher MA, Kong X, Megerian JT, Rutkove SB. Electrodiagnostic automation: principles and practice. Phys Med Rehabil Clin N Am 2005; 16:1015-32, $x$.

12. Hardy T, Sachson R, Shen S, Armbruster M, Boulton AJ. Does treatment with duloxetine for neuropathic pain impact glycemic control? Diabetes Care 2007;30:21-6.

13. Conlon CF, Rempel DM. Upper extremity mononeuropathy among engineers. J Occup Environ Med 2005;47:1276-84.

14. Armstrong T, Dale AM, Franzblau A, Evanoff BA. Risk factors for carpal tunnel syndrome and median neuropathy in a working population. J Occup Environ Med 2008;50:1355-64.

15. Leffler CT, Gozani SN, Cros D. Median neuropathy at the wrist: diagnostic utility of clinical findings and an automated electrodiagnostic device. J Occup Environ Med 2000;42:398-409.

16. Rotman MB, Enkvetchakul BV, Megerian JT, Gozani SN. Time course and predictors of median nerve conduction after carpal tunnel release. J Hand Surg Am 2004;29:367-72.

17. Elkowitz SJ, Dubin NH, Richards BE, Wilgis EF. Clinical utility of portable versus traditional electrodiagnostic testing for diagnosing, evaluating, and treating carpal tunnel syndrome. Am J Orthop (Belle Mead NJ) 2005;34:362-4.

18. Vinik AI, Emley MS, Megerian JT, Gozani SN. Median and ulnar nerve conduction measurements in patients with symptoms of diabetic peripheral neuropathy using the NC-stat system. Diabetes Technol Ther 2004;6:816-24.

19. Kong X, Gozani SN, Hayes MT, Weinberg DH. NC-stat sensory nerve conduction studies in the 
median and ulnar nerves of symptomatic patients. Clin Neurophysiol 2006;117:405-13.

20. Armstrong TN, Dale AM, Al-Lozi MT, Franzblau A, Evanoff BA. Median and ulnar nerve conduction studies at the wrist: criterion validity of the NC-stat automated device. J Occup Environ Med 2008;50: 758-64.

21. Perkins BA, Grewal J, Ng E, Ngo M, Bril V. Validation of a novel point-of-care nerve conduction device for the detection of diabetic sensorimotor polyneuropathy. Diabetes Care 2006;29:2023-7.

22. Perkins BA, Orszag A, Grewal J, Ng E, Ngo M, Bril $\mathrm{V}$. Multi-site testing with a point-of-care nerve conduction device can be used in an algorithm to diagnose diabetic sensorimotor polyneuropathy. Diabetes Care 2008;31:522-4.

23. Wells MD, Meyer AP, Emley M, Kong X, Sanchez $\mathrm{R}$, Gozani SN. Detection of lumbosacral nerve root compression with a novel composite nerve conduction measurement. Spine (Phila Pa 1976) 2002;27: 2811-9.

24. Fisher MA, Bajwa R, Somashekar KN. Lumbosarcral radiculopathties - the importance of EDX information other than needle electromyography. Electromyogr Clin Neurophysiol 2007;47(7-8):377-84.

25. Fisher MA, Bajwa R, Somashekar KN. Routine electrodiagnosis and a multiparameter technique in lumbosacral radiculopathies. Acta Neurol Scand 2008; 118:99-105.

26. Gozani SN, Kong X, Fisher MA. Factors influencing F-wave latency detection of lumbosacral root lesions using a detection theory based model. Clin Neurophysiol 2006;117:1449-57.

27. Jabre JF, Salzsieder BT, Gnemi KE. Criterion validity of the NC-stat automated nerve conduction measurement instrument. Physiol Meas 2007;28:95-104.

28. Megerian JT, Kong X, Gozani SN. Utility of nerve conduction studies for carpal tunnel syndrome by family medicine, primary care, and internal medicine physicians. J Am Board Fam Med 2007;20:60-4.

29. Kong X, Lesser EA, Potts FA, Gozani SN. Utilization of nerve conduction studies for the diagnosis of polyneuropathy in patients with diabetes: a retrospective analysis of a large patient series. J Diabetes Sci Technol 2008;2:268-74.

30. Fryback DG, Thornbury JR. The efficacy of diagnostic imaging. Med Decis Making 1991;11:88-94.

31. Guyatt GH, Tugwell PX, Feeny DH, Drummond MF, Haynes RB. The role of before-after studies of therapeutic impact in the evaluation of diagnostic technologies. J Chronic Dis 1986;39:295-304.

32. Hongo RH, Goldschlager N. Status of computerized electrocardiography. Cardiol Clin 2006;24:491-504, x.
33. Rutten FH, Kessels AG, Willems FF, Hoes AW. Electrocardiography in primary care: is it useful? Int J Cardiol 2000;74(2-3):199-205.

34. van Dijke MJ, van den Berg WN, Hoes AW. [The influence of an ECG on patient management in family practice]. Ned Tijdschr Geneeskd 19989;142: 1383-7.

35. Yawn BP, Enright PL, Lemanske RF Jr, et al. Spirometry can be done in family physicians' offices and alters clinical decisions in management of asthma and COPD. Chest 2007;132:1162-8.

36. Deeks JJ, Altman DG. Diagnostic tests 4: likelihood ratios. BMJ 2004;329:168-9.

37. Kothari MJ, Blakeslee MA, Reichwein R, Simmons Z, Logigian EL. Electrodiagnostic studies: are they useful in clinical practice? Arch Phys Med Rehabil 1998;79:1510-1.

38. Storm S, Beaver SK, Giardino N, et al. Compliance with electrodiagnostic guidelines for patients undergoing carpal tunnel release. Arch Phys Med Rehabil 2005;86:8-11, quiz 180.

39. Kendall R, Werner RA. Inter-rater reliability of the needle examination in lumbosacral radiculopathy. Muscle Nerve 2006;34:238-41.

40. Johnsen B, Fuglsang-Frederiksen A, Vingtoft S, et al. Inter- and intraobserver variation in the interpretation of electromyographic tests. Electroencephalogr Clin Neurophysiol 1995;97:432-43.

41. Tankisi H, Johnsen B, Fuglsang-Frederiksen A, et al. Variation in the classification of polyneuropathies among European physicians. Clin Neurophysiol 2003;114:496-503.

42. Salerno DF, Werner RA, Albers JW, Becker MP, Armstrong TJ, Franzblau A. Reliability of nerve conduction studies among active workers. Muscle Nerve 1999;22:1372-9.

43. Nardin RA, Rutkove SB, Raynor EM. Diagnostic accuracy of electrodiagnostic testing in the evaluation of weakness. Muscle Nerve 2002;26:201-5.

44. Kothari MJ, Preston DC, Plotkin GM, Venkatesh S, Shefner JM, Logigian EL. Electromyography: do the diagnostic ends justify the means? Arch Phys Med Rehabil 1995;76:947-9.

45. Lo JK, Finestone HM, Gilbert K, Woodbury MG. Community-based referrals for electrodiagnostic studies in patients with possible carpal tunnel syndrome: what is the diagnosis? Arch Phys Med Rehabil 2002;83:598-603.

46. Cho SC, Siao-Tick-Chong P, So Y'T. Clinical utility of electrodiagnostic consultation in suspected polyneuropathy. Muscle Nerve 2004;30:659-62. 